\title{
Patient and caregiver perspectives on virtual care: a patient- oriented qualitative study
}

\author{
Sophy Chan-Nguyen PhD, Anne O’Riordan BSc, Angela Morin BA, Lisa McAvoy MA, \\ Eun-Young Lee PhD, Veronica Lloyd MClSc, Ramana Appireddy DM MSc
}

\section{Abstract}

Background: Since the onset of the COVID-19 pandemic, virtual care solutions have been rapidly adopted across the country to provide safe, quality care to diverse patient populations. The objective of this qualitative case study was to understand patient and caregiver experiences of virtual care to identify barriers and gather suggestions to address them.

Methods: In this patient-oriented project, we sought to understand gaps in virtual care experienced by patients and caregivers, using virtual focus groups. With the assistance of a patient research liaison, we engaged 2 patient partners as full partners; they participated in study conception, data collection, data analysis and knowledge translation. Recruitment was done through email by disseminating the study poster to 30 community organizations and health units in Ontario and British Columbia. We conducted a constructivist, qualitative study guided by grounded theory methodology. One researcher employed in-vivo coding, followed by axial coding with focus group participants, followed by selective coding with the study team. The study took place from November to December 2020.

Results: We conducted 6 focus groups with 13 patients and 5 caregivers. The analysis resulted in 6 major themes and 17 minor themes. Key findings showed that barriers related to access to technology and Internet, language and cultural differences were challenges to virtual care. Participants identified special considerations surrounding caregiver and family involvement; privacy, consent and confidentiality; and the patient-physician relationship. Participants suggested that technology and the Internet be universally accessible and that virtual care modalities be integrated (e.g., consolidated patient portal) to improve virtual care.

Interpretation: There are multiple patient-identified barriers to accessing virtual care in Canada; patients can provide insights into ways to address these barriers. Future research should include robust patient engagement to explore ways to address these challenges and barriers to ensure that virtual care can be equitable, accessible and safe for all users.

Plain language summary: Although virtual care has been rapidly adopted and scaled up in health care institutions across the country, few improvements informed by patient and caregiver experiences have been made. Driven by concerns expressed by patient partners, our study team undertook a patient-partnered qualitative study to understand the barriers of virtual care from the perspectives and experiences of patients and caregivers. Our study team created the interview guide drawing from our previous patient-oriented qualitative studies and designed an orientation package to provide resources related to the focus groups and to introduce participants to the study team. Drawing from local health teams, clinics and patient advisory groups, the study team recruited 13 patients and 5 caregivers to participate in 6 focus group interviews. An analysis based on grounded theory was undertaken, with participation from both the study team and participants. Lack of access to technology or Internet and language barriers were determined to be the primary challenges in virtual care. Special considerations to caregiver and family involvement, privacy and confidentiality, as well as the patient-physician relationship were considered priorities to improving access to virtual care. Participants offered recommendations and potential solutions to address barriers and challenges in virtual care, which can serve to encourage large-scale policy and programmatic changes in patient-centred ways.

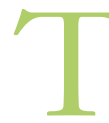
he COVID-19 pandemic has propelled virtual care into unprecedented usage in health care to provide safe alternative care. ${ }^{1-3}$ Virtual care can be defined as interactions between health care providers and patients and members of their circle of care, occurring remotely, using any forms of communication or information technologies with the aim of facilitating or maximizing quality and
Competing interests: See the end of the article.

This article has been peer reviewed.

Correspondence to: Ramana Appireddy, mrra@queensu.ca

CMAJ Open 2022 March 1. DOI:10.9778/cmajo.20210065 
effectiveness of patient care. ${ }^{4}$ In this article, the term "virtual care" specifically refers to use of video conference and telephone. Overall, virtual care is patient-centred and can enhance patient centredness in some contexts., ${ }^{5,6}$ However, digital, economic and literacy barriers can affect equal and equitable access to virtual care. ${ }^{7-10}$

Patient engagement can inform future virtual care implementation and policy. ${ }^{11}$ However, there is limited research featuring authentic patient engagement in virtual care. The objective of this study was to understand the experiences of patients and caregivers with virtual care and the barriers they face, and to gather their suggestions to address the barriers, in hopes of spurring policy and programmatic decision-makers to consider patient-oriented strategies to virtual care.

\section{Methods}

\section{Study design and setting}

We conducted a patient-oriented constructivist qualitative study using the grounded-theory approach. ${ }^{12}$ We adopted the Canadian Institutes of Health Research (CIHR) Strategy for Patient-Oriented Research (SPOR) Patient Engagement Framework, which aims to improve health care for patients and build capacity for equitable health care systems. ${ }^{11}$ The Strategy for Patient-Oriented Research increases accountability by ensuring patient involvement from conception of the project to dissemination of knowledge translation tools.

Grounded theory is a research method used to generate new understandings and theories about social processes and human behaviours using data that have been systematically collected and analyzed. ${ }^{12-15}$

We used a constructivist grounded-theory approach to gain in-depth understanding of the shared, diverging and multiple ways patients and caregivers experience barriers to virtual care. ${ }^{12} \mathrm{We}$ sought to use grounded theory because previous studies have not examined patient experiences of virtual care from patient-oriented perspectives. This perspective would provide nuanced insights into facilitators, barriers and challenges to virtual care.

We employed the constant comparative method, an inductive coding process that compares and contrasts data (e.g., thematic codes, categories and demographic characteristics) to explore variations, similarities and data differences. ${ }^{16} \mathrm{We}$ used this approach to ensure that a variety of contexts and factors were captured from the perspectives of patients and caregivers. Focus groups were chosen as the research method to conduct this qualitative study. ${ }^{17}$

The study team was based out of Queen's University, the Patient and Family Advisory Council at Kingston Health Sciences Centre and Kingston General Health Research Institute. The study was conceptualized in 2019 and was conducted virtually from November to December 2020.

Details of patient and public involvement in research are presented according to the GRIPP2 Short Form Checklist for the Reporting of Patient Engagement in Research. ${ }^{18}$ This study was reported based on the Consolidated Criteria for Reporting Qualitative Research checklist. ${ }^{19}$

\section{Participants}

Patients and caregivers from Ontario and British Columbia who were older than 18 years and willing to participate in a telephone or video conference were eligible to participate. In this study, caregiver is defined as anyone supporting the care of a loved one, friend or family member in a nonprofessional capacity. Participants without virtual care experience were also eligible to participate to identify potential barriers or challenges preventing them from engaging in virtual care.

The study team disseminated study posters and information sheets by email to local and provincial health teams, clinics and patient advocacy groups (30 entities in total) in Ontario and British Columbia over 8 weeks in October and November 2020. Consent was obtained over secure email or telephone. All participants were offered a $\$ 50$ honorarium for participating.

\section{Data collection}

The interview guide (Appendix 1, available at www.cmajopen. $\mathrm{ca} /$ content/10/1/E165/suppl/DC1) was developed in partnership between patient partners (A.O. and A.M.), the patient research liaison (L.M.), researchers (R.A., S.C-N. and E.-Y.L.) and a graduate student (V.L.). We drew on our team's previous research on virtual care to develop the guide, which went through multiple rounds of feedback and iterative revision; it was not pilot tested. $5,20,21$

We co-developed resources in compliance with the Accessibility for Ontarians with Disabilities Act to facilitate preparation and participation in virtual focus groups. These include the orientation package (Appendix 1) and Zoom tutorial (Appendix 2, available at www.cmajopen.ca/content/10/1/ E165/suppl/DC1), which were sent in advance of the focus group. The orientation package included ground rules, frequently asked questions, interview guide questions and brief biographies of study team members. An opportunity to practise or ask questions about Zoom before the scheduled focus group was offered.

The focus groups were originally planned to be conducted in person, but were modified to virtual groups to accommodate for COVID-19 restrictions. Each group included 2-4 participants and was facilitated by 2 facilitators: a patient partner (A.O.) or a patient research liaison (L.M.), and a researcher (S.C-N.). The graduate student trainee (V.L.) served as "tech support" for the duration of the focus group to assist participants with technological difficulties. In each session, the researcher shared PowerPoint slides of the questions onscreen to ensure all participants could follow along if they did not have their orientation package on hand (Appendix 3, available at www.cmajopen.ca/content/10/1/E165/suppl/ DC1). Each question was also read aloud for participants who joined by telephone. During the focus group, both facilitators took detailed notes and consolidated them at the end of each 2-hour session.

All audio was recorded and transcribed by a professional transcriber. All participants provided feedback on the conduct of the focus group and degree of patient centredness of the study using an online survey. 


\section{Patient engagement}

In January 2019, before the start of the project, 2 members of the research team (S.C.-N. and R.A.) engaged with members of the Kingston Health Sciences Centre Patient and Family Advisory Council and Kingston General Health Research Institute to identify priority areas in virtual care.

We included 2 patient partners (A.O. and A.M.) and a patient research liaison (L.M.) as part of the research team. One patient partner (A.O.) had previously worked with the research team and is familiar with issues faced by patients while accessing virtual care. ${ }^{22}$ The second patient partner (A.M.) is a patient engagement advocate. The patient research liaison (L.M.) provided guidance using best practices for meaningful and genuine patient engagement according to the CIHR SPOR framework. ${ }^{23}$ The liaison (L.M.) was formally trained in patient-oriented research with the Ontario SPOR SUPPORT Unit and has spent many years improving patient experiences in clinical trials..$^{24}$

The patient partners and patient research liaison (A.O., A.M. and L.M.) provided input on the research direction for the project, including patient engagement methods and best practices from a patient perspective, advised on the recruitment strategies, methods and format of data collection, and engaged in data collection. They also reviewed the results of the qualitative analysis and were involved in the preparation of the manuscript draft.

Researchers may enact power imbalances because they ultimately collect and represent the data. To reduce power imbalances, the researchers sought to include and prioritize voices of nonresearchers in every aspect of the research project. The study was carried out collaboratively with patient partners at all stages of the project using the consensusbuilding process. To avoid any bias, the researchers were in no way related to or involved in the care of participants. Participants were provided all details of the study team beforehand.

\section{Data analysis}

We used NVivo 10 qualitative data software for data analysis. In the first round of coding, 1 researcher (S.C-N.) independently created the coding framework using an in-vivo coding approach. Words spoken verbatim by participants were used as codes. In-vivo coding was chosen as the main analytical technique because it emphasizes by-verbatim words of the participants to ensure the coder does not deviate their analysis away from the intended meaning of the participants. ${ }^{25}$ This practice is consistent with the principles of patient-oriented research where patients should be reflected in and through research. As the study team had only 1 coder, in-vivo coding increased accountability to the data in the preliminary rounds of coding. The study team conducted a thematic analysis and sent the results to all participants who indicated their interest in participating in a validation process. This process was to ensure the themes reflected responses of participants.

In the second round of coding, axial coding was undertaken by the researcher (S.C-N.). Based on each discussion group, 6 codebooks were created with a coding tree of major and minor themes and corresponding quotes. All participants received a codebook derived from their discussion group. The study team asked 14 participants to provide feedback on the accuracy of their group's codebook based on their willingness to review the themes as indicated in an exit survey (Appendix 4, available at www.cmajopen. ca/content/10/1/E165/suppl/DC1). Six participants provided feedback on the themes, which was incorporated into the coding framework. Feedback was minor, and all participants agreed on the coding of the themes.

Based on participant feedback, the study team reviewed each codebook and collectively refined the themes. Codebooks were merged into a master codebook. In the third stage of coding, the study team used the master codebook to validate and create 3 overarching categories. The patient partners validated the master codebook to ensure that themes meaningfully captured the nuances of virtual care as well as the patient experience.

\section{Ethics approval}

All participants provided informed consent. The Queen's University Health Sciences and Affiliated Teaching Hospitals Research Ethics Board gave ethics approval for this study (DMED-2387-20).

\section{Results}

A total of 6 focus groups with 18 participants (13 patients, 5 caregivers) were conducted. No individuals dropped out of the study. Twelve participants $(67 \%)$ identified as women, 5 participants (28\%) identified as men and 1 participant $(6 \%)$ chose not to disclose their sex. The age of the participants ranged from 29 to 94 years. Each focus group lasted for 1.5 to 2 hours, with a total duration of 11.5 hours. Table 1 details the sociodemographic profiles of participants.

\section{Themes}

The analysis resulted in 6 major themes and 17 minor themes. Below we detail themes related to barriers identified by participants: access to technology and Internet barriers, language barriers and cultural differences. We then describe special considerations identified by participants regarding caregiver and family involvement; privacy, consent and confidentiality; and the patient-physician relationship. The participants made suggestions for future research directions and calls to action (major theme); we have reported these suggestions where they address points raised in the other 5 themes. Table 2 details the categories, and major and minor themes identified in the study. Table 3 presents quotes shared by the participants. We did not achieve data saturation.

\section{Barriers to virtual care}

Participants found that access to technology and Internet, language barriers and cultural differences served as barriers to virtual care. 
Table 1: Sociodemographic profile of focus group participants

\begin{tabular}{|c|c|}
\hline Characteristic & $\begin{array}{l}\text { No. }(\%)^{*} \text { of } \\
\text { participants } \\
\quad n=18\end{array}$ \\
\hline \multicolumn{2}{|l|}{ Age, yr } \\
\hline 20-39 & $7(39)$ \\
\hline $40-59$ & $2(11)$ \\
\hline $60-79$ & $8(44)$ \\
\hline $80-99$ & $1(6)$ \\
\hline \multicolumn{2}{|l|}{ Sex } \\
\hline Male & $5(28)$ \\
\hline Female & $12(67)$ \\
\hline Prefer not to answer & $1(6)$ \\
\hline \multicolumn{2}{|l|}{ Diversity } \\
\hline Visible minority & $3(17)$ \\
\hline Person with disability & $7(39)$ \\
\hline Indigenous person of Canada & $1(6)$ \\
\hline Identifies as an immigrant & $1(6)$ \\
\hline LGBTQ community & $3(17)$ \\
\hline \multicolumn{2}{|l|}{ Work status } \\
\hline Working for pay on a casual or on-call basis & $1(6)$ \\
\hline Not in labour force, unable to work & $1(6)$ \\
\hline Retired & $9(50)$ \\
\hline Student (includes students working part-time) & $4(22)$ \\
\hline Homemaker & $1(6)$ \\
\hline Prefer not to answer & $2(11)$ \\
\hline \multicolumn{2}{|l|}{ Education } \\
\hline Completed high school & $1(6)$ \\
\hline $\begin{array}{l}\text { Some postsecondary education (college, } \\
\text { university, technical training) }\end{array}$ & $3(16)$ \\
\hline Completed college & $4(22)$ \\
\hline Completed technical training (e.g., apprenticeship) & $1(6)$ \\
\hline Completed university & $4(22)$ \\
\hline $\begin{array}{l}\text { Completed postgraduate, professional or } \\
\text { graduate degree }\end{array}$ & $5(28)$ \\
\hline \multicolumn{2}{|l|}{ Perspective } \\
\hline Patient, patient advisor or patient partner† & $13(72)$ \\
\hline Family member or caregiver & $5(28)$ \\
\hline \multicolumn{2}{|l|}{ Geography } \\
\hline Ontario & $2(11)$ \\
\hline British Columbia & $16(89)$ \\
\hline \multicolumn{2}{|l|}{ Experience with virtual care } \\
\hline Participants with experience & $17(94)$ \\
\hline Participants without experience & $1(6)$ \\
\hline \multicolumn{2}{|c|}{$\begin{array}{l}\text { Note: LGBTQ = lesbian, gay, bisexual, transgender, queer or questioning. } \\
{ }^{*} \text { The percentages in some categories may sum to more than } 100 \text { because of } \\
\text { rounding. } \\
\text { †Patient advisors and patient partners who participated in this study do not } \\
\text { include the patient partners or patient research liaison on the research team. }\end{array}$} \\
\hline
\end{tabular}

\section{Access to technology and Internet}

One of the most reported barriers to virtual care was limited access to technology or the Internet. This was of particular concern for rural residents and aging individuals. Participants warned against making assumptions that everyone can adopt and use digital health technologies to receive virtual care. Participants asserted that universal access to Internet would need to be made readily accessible if virtual care were to become a standardized option of care for all Canadians moving forward.

\section{Language barriers and cultural differences}

Participants expressed that a lack of proficiency in English or French may pose a barrier to successful virtual care for recent immigrants. Participants drew from experiences helping their parents, siblings and others in their social circles in expressing this concern. Participants also expressed that knowledge and understanding of patients' rights and preferences can help to relieve some difficulties associated with cultural differences.

\section{Special considerations for virtual care}

Three major themes were identified related to special considerations in accessing and receiving successful virtual care: caregiver and family involvement, privacy and consent, and the physician-patient relationship.

\section{Caregiver and family involvement}

Participants noted caregivers can play a crucial role in accessing and experiencing successful virtual care for some patients, and caregivers' own digital and technological literacy can affect it.

One caregiver recalled that they were asked to provide information about clinical symptoms and assess their dependent, which they were unprepared to do. Patients and caregivers agreed that caregivers could be given additional responsibilities in virtual care, adding to caregiver burden. They suggested the health care provider should assess the caregiver's comfort level in participating in virtual care sessions and offer supports where needed. Participants also suggested that an integrated approach with data sharing between health care platforms and organizations could enhance overall user experience with virtual care, reduce caregiver burden and, ultimately, improve patient outcomes.

\section{Privacy, consent and confidentiality considerations}

Participants expressed the importance of patient autonomy in virtual care decision-making as well as the need for patient and caregiver confidentiality. Health care providers need to be aware of constraints placed on patients and caregivers to discuss confidential information because of lack of privacy during virtual care encounters; this may result in additional stress and caregiver burden. Participants also expressed the need for obtaining patient input and consent for presence of additional parties during a virtual care session.

\section{Patient-physician relationship}

Some participants reported their interactions with their health care provider felt "mechanical" through virtual care. Participants reported having an "artificial feeling" and that 


\begin{tabular}{|ll|}
\hline Table 2: Major and minor themes derived from the master codebook \\
\hline Categories and major themes & \multicolumn{1}{c|}{ Minor themes } \\
\hline Category: common barriers to virtual care \\
\hline $\begin{array}{l}\text { Access to technology and Internet } \\
\text { are barriers }\end{array}$ & $\begin{array}{l}\text { - No or limited access to technology or Internet } \\
\text { - Rapid technology changes challenging and overwhelming } \\
\text { - Digital literacy can affect virtual care access }\end{array}$ \\
\hline $\begin{array}{ll}\text { Language barriers and cultural } \\
\text { differences can affect virtual care }\end{array}$ & $\begin{array}{l}\text { - Inability to express concerns } \\
\text { - Cultural barriers could affect knowledge of virtual care }\end{array}$ \\
\hline Category: special considerations improve patient and caregiver experiences with virtual care
\end{tabular}

certain modalities, such as email, were replacing the desirable face-to-face component. Participants also expressed concern about the attentiveness of their provider during these sessions. Some participants felt it was difficult to interrupt the provider to ask for clarification or convey concerns during a session compared with in-person interactions. Participants felt it was challenging to establish a personal connection with their provider.

\section{Interpretation}

In our study, the participating patients and caregivers identified digital literacy, technology ownership, Internet access, lack of proficiency in English and new immigrant status as potential barriers to virtual care access and success. Participants also voiced important concerns around privacy, confidentiality, caregiver involvement, caregiver burden and patient-physician relationship in the context of virtual care.

Our study adds to the growing body of literature detailing the barriers of virtual care and special considerations that must be undertaken to ensure that it remains a patientcentred practice. ${ }^{29,30}$ Our study found 2 prominent barriers to virtual care - lack of access to technology or Internet, and language barriers or cultural differences. These challenges perhaps point to the way traditional social determinants of health can influence health outcomes, including virtual care access and uptake. ${ }^{31,32}$ Some determinants of health, such as illiteracy, can serve as both a barrier to technology use and a language barrier, amplifying the challenges to access to virtual care; health care providers should be aware of this. ${ }^{33}$

Special consideration must also be paid to the ways virtual care may change relationships between patients, caregivers and providers. A study examining the implementation of a virtual diabetes consultation program found patients may be wary of having a virtual discussion about distress brought on by their health conditions. ${ }^{10}$ Our study echoes these concerns, as some participants felt less engaged or connected with their health care provider within the virtual care appointment. Understanding a patient's preference for virtual versus inperson care and finding ways to establish rapport in an interaction may alleviate some of the patient's concerns. Providers should always obtain consent from patients privately and should confirm consent if someone else is joining the session.

Our study found that caregivers may take on a disproportionate responsibility in assisting with a virtual care session, adding to the burden of care they may already experience through caregiving. Providers should seek to understand the extent of a caregiver's responsibilities and offer support whenever possible.

Involving patients in virtual care research can be a meaningful way to address some of the issues explored in this article. Patients can provide unique solutions because of their vantage point as recipients of virtual care. ${ }^{34}$ Improved access to Internet and integrated patient portals were identified as 2 recommendations that could improve patient experiences 
Table 3: Selected quotes from participants according to the discussion themes

\begin{tabular}{|ll}
\hline Major themes & \multicolumn{1}{c}{ Quotes } \\
\hline $\begin{array}{l}\text { Access to technology and } \\
\text { Internet are barriers }\end{array}$ & "I was speaking with one patient who said that she can't afford Internet so she gets in her wheelchair \\
and goes to the library and sits outside the library and picks up their Internet. But during the pandemic \\
when they closed the library they also shut down the Internet." (Group 4, Caregiver 1) \\
"My husband had 2 strokes and both of them were during the pandemic, so everything was done \\
through Zoom, which we'd never used before. He is not computer literate and he wouldn't really be \\
there for a lot of the discussions. Physio, how do you do physio over Zoom? That's pretty tricky. OT and \\
speech were all done by Zoom. But after about 10 minutes we kind of lost his interest, so it was really \\
hard to keep him involved." (Group 4, Caregiver 2) \\
"For the first one, income, like I said, I don't own a computer myself so I can't afford a computer right \\
now, especially with COVID times and not working so I'm sure l'm not the only one in that boat right \\
now." (Group 6, Caregiver 1) \\
"The thing about virtual care would be so great, but you guys are talking about in your room being a \\
place where there's a dead zone; we've got whole roads that are dead zones." (Group 5, Patient 1)
\end{tabular}

Language barriers and cultural differences can affect virtual care
"English is not my mother tongue and then I just remember one of my appointment with a doctor. It was about my shoulder, so even though I have a degree in physical education, I got to know all the muscles in Latin, I was unable to explain my problem in English." (Group 5, Patient 2)

"I mean, in my family, my parents don't speak English very well, so it happens that I often have to call back and just get them clarification on some of the results or anything that's happened at their appointments or my sibling's appointments." (Group 1, Patient 3)

Caregiver and family involvement

"My husband can't use a computer. He has no idea even how to turn it on. All the appointments have to be set up when I'm there. He can't answer the phone, he has no strength in his hands, so I have to make all the arrangements to get the phone and get it on speaker and it's really difficult for him to deal with technology." (Group 4, Caregiver 2)

"It's impacted my mother because she doesn't own a cellphone and she doesn't have Wi-Fi or a computer. [...] I feel like I need to be there all the time. I'm only 2 blocks away from my mom, but we speak quite a few times during the day and I just feel like I need to be around all the time, yeah." (Group 6, Caregiver 1)

"You might not have access to virtual care and it would be up to the doctor to realize that you live separately. The caregiver may have access to a computer and Internet, but the patient may not. Does that mean the caregiver has to go and take all their phone or whatever over to the patient every time? There's a lot of moving parts there that you need to think about." (Group 4, Caregiver 1)

Privacy, consent and confidentiality considerations

"I think the issue of privacy comes up to my mind and I put something in the chat about that as well just, if you're at home and you have other family at home and maybe you want to have a private conversation, your family might listen in and you don't want them to. That impacts things." (Group 3, Patient 1)

"But also I was thinking about privacy, what if the person doesn't want to share some of private ... it doesn't have to be private but your health is part of your privacy. So you may not want to share certain parts of your health issues with whoever the person is next to you. So it's sort of ... I don't know, creates a sort of tension where privacy is questioned 'cause if I say I don't want you to be present, but we are in a partnership, why don't you want me to hear this, etc." (Group 1, Caregiver 1)

Patient-physician relationship "There was kind of a little bit of an almost artificial, like you're talking to somebody but you don't really get that they're real and whatever 'cause you've not seen them face-to-face, so there was some of that but the care was, in all but one instance, was very good." (Group 1, Caregiver 2)

"Lastly, when I need to talk to my GP I email her and that seems to work, although I have a sense that emailing is replacing face-to-face contact and I'm not so happy about the future of that." (Group 2 , Patient 2)

“I don't know, it's just an assumption ‘cause you don't see the person and then another thing, you don't know if the person is talking to you attentively. They might be doing something on computer and then talking to you on the side, so there's always this hesitant ... I don't know." (Group 5, Patient 2)

"I found too that when doctors know that you're actually online for the kids and for yourself, I found that it's hard to find yourself sometimes feeling weak and feeling scared in appointments especially online, but still have to advocate. I find it easier to advocate in person and say, you know, this needs to be done, or feeling weak in person, but then you have to find yourself in between to find any support." (Group 4, Caregiver 3)

Future research directions or calls to action
"I think when things are integrated it makes my life a whole lot easier. The service that I mentioned when I have tried video conferencing I really liked that I could see the doctor's notes and that I could specify the pharmacy of my choice and it would be sent. I think that's really helpful to have all those components put together and not have all these different parts that you have to follow up on." (Group 3, Patient 3) "I think the technical side has to develop so it becomes universally accessible to the physicians and patients in an efficient way." (Group 2, Patient 1)

Note: GP = general practitioner, OT = occupational therapy. 
with virtual care. Further patient-oriented research in these areas could address key challenges related to access to virtual care for Canadians.

The rapid implementation of virtual care can pose challenges to both providers and patients unprepared to use or provide it because of lack of access or familiarity. ${ }^{27}$ Such concerns have strong implications for patient safety and access to equitable care in Canada. ${ }^{9,28}$ Data are limited on challenges and barriers in accessing virtual care among the general population and among those who are vulnerable and affected by unfavourable social determinants of health profiles. Future studies should focus on barriers to virtual care access and ways to address them.

\section{Limitations}

Some limitations of our study include limited enrolment of participants with diverse socioeconomic status, health conditions and experiences, and limited sampling of older adults (especially age $>80 \mathrm{yr}$ ). Despite our efforts to recruit from more than 30 local agencies, health units and organizations, we were unable to increase the sample size of our study. COVID-19 pandemic restrictions limited our ability to meet and conduct community outreach activities, thus limiting us to online recruitment. The virtual focus groups also limited the number of participants in a session for meaningful engagement. Overall, these factors influenced the sociodemographic profile of participants, possibly resulting in lack of data saturation. Failure to achieve data saturation could be attributed to the difficulty of recruiting patients when virtual care was still relatively nascent. However, the major themes found in this study were reiterated by every focus group within the study.

One individual recruited to the study did not have experience with virtual care. However, their experiences were consistent with some of the major challenges identified by those who had used virtual care. Limitations inherent to focus groups include bias or influence that may be introduced by dominant members of a group, experiences that only reflect the sample comprising the group, hesitation to answer questions because of peer pressure and potential for moderator bias.

\section{Lessons learned from patient engagement}

Patients can increase scientific rigour by offering perspectives leading to establishment of relevant research goals, methodologies, interpretations and research outcomes. ${ }^{26}$ The power of patient engagement in research is in the combination of methods used to hear patient perspectives in the project. Patient partners were included from the outset as full team members and were involved in every stage of the research to ensure that the project proceeded with a substantial and consistent patient-oriented lens.

Communication and strong relationship building are key to the success of patient engagement in research. We learned how much language matters. Words can take on different interpretations depending on the context from which different team members come, especially for patient-partner team members. Careful attention and reflection about communication were essential to avoid misinterpretation, particularly in the context of the virtual world in which this research was completed. Developing a level of trust, understanding and collaboration enabled frank discussion with openness to challenge assumptions. All team members felt safe to question and share ideas. Having patient partners on the project team helped to identify potential points of confusion before beginning the project.

Having clear objectives, defining the project scope along with expectations and deliverables at the start is important. The team found the scope of the project changed during team discussions and attributed this to the diverse perspectives being heard. Creative thinking was encouraged, and meetings often ended with far more ideas to consider and questions to address than when the meeting began. Patient partners often challenge the status quo and in so doing help to create a dynamic that embraces those challenges. New points were continually being raised from the variety of perspectives within the team, leading to more fluidity than structure within the project. Once the scope of a project had been defined, it was important to identify and list potential new projects that extended beyond the predefined scope and to be cognizant of those parameters when new issues arose. The team learned that patient-oriented research is a dynamic process, with opportunities to embrace new ideas and the responsibility to maintain a focus on mutually identified goals and objectives.

\section{Conclusion}

In this qualitative study co-designed by patients, we examined barriers in accessing virtual care from a patient and caregiver perspective in Canada. We consider this research a timely contribution to the growing body of literature promoting the benefits of virtual care, particularly in the midst of the COVID-19 pandemic. Our findings confirm the presence of important barriers and the need to include patients and caregivers during virtual care implementation to mitigate them. We hope our study encourages other researchers and practitioners to include patient voices and consider nuances of patient engagement and experiences within virtual care.

\section{References}

1. Dewar S, Lee PG, Suh TT, et al. Uptake of virtual visits in a geriatric primary care clinic during the COVID-19 pandemic. 7 Am Geriatr Soc 2020;68: 1392-4.

2. Webster P. Virtual healthcare in the era of COVID-19. Lancet 2020;395: 1180-1.

3. Hollander JE, Carr BG. Virtually perfect? Telemedicine for COVID-19. N Engl 7 Med 2020;382:1679-81.

4. Shaw J, Jamieson T, Agarwal P, et al. Virtual care policy recommendations for patient-centred primary care: findings of a consensus policy dialogue using a nominal group technique. 7 Telemed Telecare 2018;24:608-15.

5. Chan S, O'Riordan A, Appireddy R. Exploring the determinants and experiences of senior stroke patients with virtual care. Can 7 Neurol Sci 2021;48:87-93.

6. Danilewitz M, Ainsworth NJ, Bahji A, et al. Virtual psychiatric care for older adults in the age of COVID-19: challenges and opportunities. Int 7 Geriatr Psychiatry 2020;35:1468-9.

7. Hardcastle L, Ogbogu U. Virtual care: Enhancing access or harming care? Healthc Manage Forum 2020;33:288-92.

8. Cukier A. 'Left out of this equation': Why virtual healthcare is leaving some Ontarians behind. TVO 2020 Nov. 17. Available: https://www.tvo.org/article/ left-out-of-this-equation-why-virtual-health-care-is-leaving-some-ontarians -behind (accessed 2021 Aug. 16). 
9. Crawford A, Serhal E. Digital health equity and COVID-19: the innovation curve cannot reinforce the social gradient of health. 7 Med Internet Res 2020; 22:e19361.

10. Kilvert A, Wilmot EG, Davies M, et al. Virtual consultations: Are we missing anything? Pract Diabetes 2020;37:143-6.

11. Strategy for Patient-Oriented Research (SPOR): putting patients first. Ottawa: Canadian Institutes of Health Research; modified 2019 May 27.

12. Charmaz K. Constructing grounded theory. 2nd ed. London (UK): SAGE Publications; 2014.

13. Ivey J. What is grounded theory? Pediatr Nurs 2017;43:288-308.

14. Oktay JS. Grounded theory. Oxford (UK): Oxford University Press; 2012.

15. Noble H, Mitchell G. What is grounded theory? Evid Based Nurs 2016;19:34-5.

16. Hallberg LR-M. The "core category" of grounded theory: making constant comparisons. Int 7 Qual Stud Health Well-being 2006;1:141-8.

17. Hennink MM. Focus group discussions. New York: Oxford University Press; 2014.

18. Staniszewska S, Brett J, Simera I, et al. GRIPP2 reporting checklists: tools to improve reporting of patient and public involvement in research. BMF 2017; 358:j3453.

19. Tong A, Sainsbury P, Craig J. Consolidated criteria for reporting qualitative research (COREQ): a 32 -item checklist for interviews and focus groups. Int 7 Qual Health Care 2007;19:349-57.

20. Saad M, Chan S, Nguyen L, et al. A qualitative examination of patient experiences and determinants of virtual postnatal follow-up visits. BMC Pregnancy and Childbirth 2021;21:543

21. Chan-Nguyen S, Karacam M, Ritsma B, et al. Patient perceptions on the virtual amyotrophic lateral sclerosis clinic during COVID-19. Can 7 Neurol Sci 2021 July 13 [Epub ahead of print]. doi: 10.1017/cjn.2021.160.

22. Chan S, O'Riordan A, Appireddy R. Exploring the determinants and experiences of senior stroke patients with virtual care. Can 7 Neurol Sci 2021;48: $87-93$.

23. IAP2 core values. IAP2. Available: https://www.iap2 canada.ca/Resources/ Documents/0702-Foundations-Core-Values-MW-rev1.pdf (accessed 2021 Aug. 16).

24. Patient engagement in research: catalogue of organizations in Ontario. Toronto: Ontario SPOR SUPPORT Unit; 2019.

25. Given LM, editor. The SAGE encyclopedia of qualitative research methods. Thousand Oaks (CA): SAGE Publications; 2008.

26. Flowers M, Gunderson J, Hall D. Evaluating the patient family advisor experience in patient-oriented research. Healthc Q 2020;23:28-33.

27. Zachrison KS, Yan Z, Sequist T, et al. Patient characteristics associated with the successful transition to virtual care: lessons learned from the first million patients. 7 Telemed Telecare 2021 June 13 [Epub ahead of print]. doi: $10.1177 / 1357633$ X211015547.

28. Leovic MP, Robbins HN, Foley MR, et al. The "virtual" obstetrical intensive care unit: providing critical care for contemporary obstetrics in nontraditional locations. Am 7 Obstet Gynecol 2016;215:736.e1-e4.

29. Sanderson KE, Spithoff KD, Corovic M, et al. An evaluation of cardiology virtual care during the COVID-19 pandemic. CFC Open 2021 May 31 [Epub ahead of print]. doi: 10.1016/j.cjco.2021.05.015.

30. Hyde AM, Watt M, Carbonneau M, et al. Understanding preferences toward virtual care: a pre-COVID mixed methods study exploring the perspectives of patients with chronic liver disease. Telemed 7 E Health 2021 June 3 [Epub ahead of print]. doi: 10.1089/tmj.2021.0099.
31. Bambra C, Gibson M, Sowden A, et al. Tackling the wider social determinants of health and health inequalities: evidence from systematic reviews. 7 Epidemiol Community Health 2010;64:284-91.

32. McGibbon E, Etowa J, McPherson C. Health-care access as a social determinant of health. Can Nurse 2008;104:22-7.

33. Onasch J. Improving digital literacy in hospitals. Madison (WI): Revelation; 2018. Available: https://revelation.agency/improving-digital-literacy-in-hospitals/ (accessed 2021 Aug. 16).

34. Shaw J, Jamieson T, Agarwal P, et al. Virtual care policy recommendations for patient-centred primary care: findings of a consensus policy dialogue using a nominal group technique. 7 Telemed Telecare 2018;24:608-15.

Competing interests: Ramana Appireddy reports support from the Canadian Institutes of Health Research (CIHR, project \# 432988) and Canada Health Infoway for the present manuscript, and research grants from CIHR, PSI Foundation, Canada Health Infoway, Department of Medicine, Queen's University, Southeastern Ontario Academic Medical Organization, and Kingston General Hospital Research Institute. No other competing interests were declared.

Affiliations: Department of Family Medicine (Chan-Nguyen), Queen's University; Patient and Family Advisory Council (O'Riordan, Morin), and Patient and Family Centred Care (Morin), and Kingston General Health Research Institute (McAvoy, Lloyd), Kingston Health Sciences Centre; School of Kinesiology and Health Studies (Lee), and Department of Medicine (Appireddy), Queen's University, Kingston, Ont.

Contributors: All authors contributed to the conception of the study and study design. Sophy Chan-Nguyen, Anne O'Riordan, Lisa McAvoy and Veronica Lloyd contributed to data collection. All authors contributed to data analysis and interpretation of data. Sophy Chan-Nguyen drafted the manuscript, which the other authors revised. All authors gave final approval of the version to be published and agree to be accountable for all aspects of the work.

Funding: This project was supported by the Canadian Institutes of Health Research (Project No. 432988).

Content licence: This is an Open Access article distributed in accordance with the terms of the Creative Commons Attribution (CC BY-NC-ND 4.0) licence, which permits use, distribution and reproduction in any medium, provided that the original publication is properly cited, the use is noncommercial (i.e., research or educational use), and no modifications or adaptations are made. See: https://creativecommons.org/licenses/by-nc-nd/4.0/

Data sharing: In accordance with the Queen's University Health Sciences and Affiliated Teaching Hospitals Research Ethics Board, only members of the research team have access to the data.

Supplemental information: For reviewer comments and the original submission of this manuscript, please see www.cmajopen.ca/content /10/1/E165/supp1/DC1. 